1 Hacettepe Journal of Mathematics and Statistics

$\bigcap$ Volume 47 (6) (2018), 1453-1466

\title{
On the weak solutions and determining modes of the g-Bénard problem
}

\author{
Muharrem Özlük* ${ }^{* \dagger \ddagger}$ and Meryem Kaya ${ }^{\ddagger}$
}

\begin{abstract}
In this paper we study the existence and uniqueness of weak solutions of the g-Bénard problem. Then, we investigate the long-term dynamics; specifically, we derive upper bounds for the number of determining modes for this system.
\end{abstract}

Keywords: Bénard convection, existence and uniqueness of weak solutions, determining modes.

Mathematics Subject Classification (2010): 35B40, 35D30, 35Q35.

Received : 29.02.2016 Accepted : 28.05.2016 Doi : 10.15672/HJMS.20174622762

\section{Introduction}

In the field of the fluid dynamics the Boussinesq equations is studied and since the fluid on the earth is laid in a layer we consider the Boussinesq equations in bounded domains.

Let $\Omega_{g}$ be a bounded domain defined by

$$
\Omega_{g}=\left\{\left(y_{1}, y_{2}, y_{3}\right) \in \mathbb{R}^{3}:\left(y_{1}, y_{2}\right) \in \Omega_{2}, 0<y_{3}<g\right\}
$$

where $\Omega_{2}$ is a bounded region in the plane and $g=g\left(y_{1}, y_{2}\right)$ is a smooth function defined on $\Omega_{2}$. The governing equations for the fluid are of the form

$$
\begin{aligned}
\frac{\partial U}{\partial t}+(U \cdot \nabla) U-\nu \Delta U+\nabla P & =\xi \Theta+F_{1}(t) \\
\nabla \cdot U & =0 \\
\frac{\partial \Theta}{\partial t}+(U \cdot \nabla) \Theta-\kappa \Delta \Theta & =F_{2}(t)
\end{aligned}
$$

*Batman University, Department of Mathematics, 72060, Batman, Turkey, Email: muharrem.ozluk@gmail.com

$\dagger_{\text {Corresponding Author. }}$

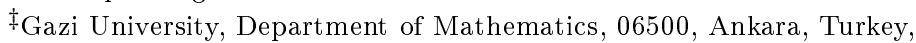
Email: meryemk@gazi.edu.tr 
where $U=\left(U_{1}, U_{2}, U_{3}\right)$ is the fluid velocity, $P$ is the pressure, $\Theta$ is the temperature, $F_{1}$ is the external force function, $F_{2}$ is the heat source function, $\xi \in \mathbb{R}^{3}$ is a constant vector, $\nu$ kinematic viscosity and $\kappa$ thermal diffusivity are positive constants.

The system of equations $(1.1)-(1.3)$ describes the large scale motion of the fluids [7]. The existence and uniqueness of the weak solution of the Boussinesq equations has been studied for arbitrary dimension, (see $[30,21]$ ). The Navier - Stokes equations on thin domains studied by Hale and Raugel [9]-[11] concerning the reaction - diffusion and damped wave equations on thin domains. Then, Raugel and Sell [33, 34] proved global existence of strong solutions for large initial data and forcing terms in thin three-dimensional domains for the purely periodic boundary conditions and the periodic-Dirichlet boundary conditions. By averaging along the vertical direction and using the uniqueness of solutions of two-dimensional Navier-Stokes equations, Temam and Ziane obtained limit models, together with existence and global regularity results in [40, 41]. One can refer [12]-[18],[28],[29] for more on thin domain problems. The existence of weak solutions of the g-Navier-Stokes equations established by Roh [35]-[37] for periodic boundary conditions as well as Dirichlet boundary conditions on bounded domains. Kaya and Çelebi [25] discussed the existence and uniqueness of weak solutions of g-Kelvin - Voight equations by the use of the well known Feado - Galerkin method. Also they established the global existence and uniqueness of weak solutions of the regularized Bénard problem and give a proof for the existence of global attractor in the three-dimensional case [26]. The asymptotic behaviour of weak solutions for the three-dimensional Bénard problem is studied in [22] and they constructed a one parameter family of multivalued semiflows. Also, the existence of global attractors in the weak topology for the 3D Bénard system in bounded domains was proved in [24] and the existence of global $\varphi$-attractor for a modified 3D Bénard system on channel-like domains in [23].

The article is organized as follows. In Section 2, we introduce some notations and the functional setting of the Bénard problem. In Section 3, we prove the existence and uniqueness of weak solutions of the g-Bénard problem. In Section 4, we estimate upper bounds for the number of determining modes for the g-Bénard problem.

\section{Preliminaries}

We introduce the usual notation used in the context of the mathematical theory of Navier-Stokes equations [38, 39]. Let $\Omega_{g}=\Omega_{2} \times(0, g)=(0,1) \times(0,1) \times(0, g)$ where $g=g\left(y_{1}, y_{2}\right)$ is a smooth function defined on $\Omega_{2}$. In addition we assume that

$$
\begin{array}{r}
0<m_{0} \leq g\left(y_{1}, y_{2}\right) \leq M_{0} \text { for all }\left(y_{1}, y_{2}\right) \in \Omega_{2} \\
|\nabla g|_{\infty}=\sup _{\Omega_{2}}|\nabla g|<\infty, \quad g \in C_{p e r}^{\infty}\left(\Omega_{2}\right) .
\end{array}
$$

We consider the three-dimensional Bénard convection model under Boussinesq approximation for incompressible fluids on $\Omega_{g}$ with periodic boundary conditions

$$
\begin{aligned}
\frac{\partial U}{\partial t}+(U \cdot \nabla) U-\nu \Delta U+\nabla P & =\xi \Theta+F_{1}(t), & & \Omega_{g} \times[0, T] \\
\nabla \cdot U & =0, & & \Omega_{g} \times[0, T] \\
\frac{\partial \Theta}{\partial t}+(U \cdot \nabla) \Theta-\kappa \Delta \Theta & =F_{2}(t), & & \Omega_{g} \times[0, T]
\end{aligned}
$$

We assume that $U$ and $\Theta$ satisfy the boundary conditions

$$
\begin{gathered}
U \cdot n=0, \quad \nabla \Theta \cdot n=0 \quad \text { on } \Gamma_{1} \cup \Gamma_{2} \\
\left.\Theta\right|_{y_{3}=g}=0,\left.\quad \frac{\partial \Theta}{\partial y_{3}}\right|_{y_{3}=g}=0
\end{gathered}
$$


where $n$ is the outward unit normal vector and

$$
\begin{aligned}
& \Gamma_{1}=\left\{\left(y_{1}, y_{2}, y_{3}\right) \in \Omega_{g}: y_{3}=0\right\} \\
& \Gamma_{2}=\left\{\left(y_{1}, y_{2}, y_{3}\right) \in \Omega_{g}: y_{3}=g\left(y_{1}, y_{2}\right)\right\}
\end{aligned}
$$

For the initial conditions we consider $U(y, 0)=U_{0}(y)$ and $\Theta(y, 0)=\Theta_{0}(y)$ defined in $\Omega_{g}$ and for the external functions $F_{1}$ and $F_{2}$, we will require that

$$
\int_{\Omega_{g}} U_{0}(y) d y=0, \quad \int_{\Omega_{g}} \Theta_{0}(y) d y=0, \quad \int_{\Omega_{g}} F_{1} d y=0, \quad \int_{\Omega_{g}} F_{2} d y=0 .
$$

The approach we use is an adaptation of the theory presented in [33, 34, 35]. The change of variables,

$$
x_{1}=y_{1}, x_{2}=y_{2} \text { and } x_{3}=\frac{1}{g\left(y_{1}, y_{2}\right)} y_{3}
$$

maps $\Omega_{3}$ onto $\Omega_{g}$ where $\Omega_{3}=\Omega_{2} \times(0,1)$. Then for $U=\left(U_{1}\left(y_{1}, y_{2}\right), U_{2}\left(y_{1}, y_{2}\right), U_{3}\left(y_{1}, y_{2}, y_{3}\right)\right)=$ $u\left(x_{1}, x_{2}, x_{3}\right)$ similarly in [35] we obtain from the boundary conditions of the problem that

$$
u_{3}=-g x_{3}\left(\frac{\partial u_{1}}{\partial x_{1}}+\frac{\partial u_{2}}{\partial x_{2}}\right) .
$$

The orthogonal average operators $M$ and $\tilde{M}$ in the thin direction are in the following;

$$
\begin{aligned}
(M \varphi)\left(y_{1}, y_{2}\right) & =\frac{1}{g} \int_{0}^{g} \varphi\left(y_{1}, y_{2}, y_{3}\right) d y_{3}, & & \varphi \in L^{2}(\Omega, g) \\
\tilde{M} u & =\left(M u_{1}, M u_{2}, 0\right), & & u \in\left(L^{2}(\Omega, g)\right)^{3} .
\end{aligned}
$$

Let $v=\left(v_{1}, v_{2}\right)$ defined by $v_{i}=M u_{i}=\int_{0}^{1} u_{i}\left(x_{1}, x_{2}, x_{3}\right) d x_{3}, i=1,2$. Then we have $v_{1}=u_{1}, \quad v_{2}=u_{2}$ and from the incompressibility condition $(1.2)$ we have $\nabla \cdot(g v)=0$. Therefore, (see, $[35,1]$ )

$$
u_{3}\left(x_{1}, x_{2}, x_{3}\right)=x_{3}\left(\frac{\partial g}{\partial x_{1}} v_{1}+\frac{\partial g}{\partial x_{2}} v_{2}\right)
$$

The application of the averaging operators to the system (1.1) - (1.3) results in an involving system of equations. In order to simplify this system we impose the boundary conditions $(2.2)-(2.3)$. Therefore we arrive at the system

$$
\begin{array}{rr}
\frac{\partial v}{\partial t}+(v \cdot \nabla) v-\nu \Delta v+M \nabla p=\xi \phi+M f_{1}, & \Omega_{2} \times[0, T] \\
\nabla \cdot(g v)=0, & \Omega_{2} \times[0, T] \\
\frac{\partial \phi}{\partial t}+(v \cdot \nabla) \phi-\kappa \Delta \phi-\frac{2 \kappa}{g}(\nabla g \cdot \nabla) \phi-\frac{\kappa \Delta g}{g} \phi=M f_{2}, & \Omega_{2} \times[0, T]
\end{array}
$$

where $\phi=M \theta$.

$L^{2}(\Omega, g)$ denotes the Hilbert space with the inner product $\langle u, v\rangle_{g}=\int_{\Omega}(u \cdot v) g d x$, the norm $|u|_{g}^{2}=\langle u, u\rangle_{g}$. Similarly $H^{1}(\Omega, g)$ is the space with the norm

$$
|u|_{H^{1}(\Omega, g)}^{2}=\langle u, u\rangle_{g}+\sum_{i=1}^{n}\left\langle\frac{\partial u}{\partial x_{i}}, \frac{\partial u}{\partial x_{i}}\right\rangle_{g} .
$$


We define

$$
\begin{aligned}
\mathcal{V}_{1} & =\left\{u \in\left(C_{\text {per }}^{\infty}(\Omega)\right)^{n}: \nabla \cdot(g u)=0, \int_{\Omega} u d x=0 \text { on } \Omega\right\} \\
H_{g} & =\text { the closure of } \mathcal{V}_{1} \text { in } L^{2}(\Omega, g) \\
V_{g} & =\text { the closure of } \mathcal{V}_{1} \text { in } H^{1}(\Omega, g) \\
V_{g}^{\prime} & =\text { the dual space of } V_{g} \\
V_{2} & =\left\{\varphi \in C_{p e r}^{\infty}(\Omega): \int_{\Omega} \varphi d x=0\right\} \\
W_{g} & =\text { the closure of } \mathcal{V}_{2} \text { in } H^{1}(\Omega, g) \\
W_{g}^{\prime} & =\text { the dual space of } W_{g} \\
Q & =\text { the closure of }\left\{\nabla \varphi: \varphi \in C_{p e r}^{1}(\bar{\Omega}, R)\right\} \text { in } L^{2}(\Omega) .
\end{aligned}
$$

where $H_{g}$ is endowed with the inner product and the norm in $L^{2}(\Omega, g)$ and $V_{g}, W_{g}$ are endowed with the inner product and the norm in $H^{1}(\Omega, g)$. The inclusions

$$
V_{g} \subset H_{g}=H_{g}^{\prime} \subset V_{g}^{\prime}, \quad W_{g} \subset L^{2}(\Omega, g) \subset W_{g}^{\prime}
$$

are valid where each space is dense in the following one and the injections are continuous $[30,35]$. By the Riesz representation theorem, it is possible to write

$$
\langle f, u\rangle_{g}=(f, u)_{g}, \quad \forall f \in H_{g}, \forall u \in V_{g} .
$$

Also, we define the orthogonal projection $P_{g}$ as $P_{g}: L_{p e r}^{2}(\Omega, g) \rightarrow H_{g}$ and we have that $Q \subseteq H_{g}^{\perp}$. Similarly, we define $\tilde{P}_{g}$ as $\tilde{P}_{g}: L_{p e r}^{2}(\Omega, g) \rightarrow W_{g}$. By taking into account the following equality,

$$
-\frac{1}{g}(\nabla \cdot g \nabla u)=-\Delta u-\frac{1}{g}(\nabla g \cdot \nabla) u
$$

we define the g-Laplace operator and g-Stokes operator as $-\Delta_{g} u=-\frac{1}{g}(\nabla \cdot g \nabla u)$ and $A_{g} u=P_{g}\left[-\Delta_{g} u\right]$ respectively.

2.1. Proposition. [35, 1] For the g-Stokes operator $A_{g}$, the followings hold:

(1) The g-Stokes operator $A_{g}$ is a positive, self-adjoint operator with compact inverse, where the domain of $A_{g}$ is $D\left(A_{g}\right)=V_{g} \cap H^{2}(\Omega, g)$.

(2) There exist countable eigenvalues of $A_{g}$ satisfying

$$
0<\frac{4 \pi^{2} m_{0}}{M_{0}} \leq \lambda_{1} \leq \lambda_{2} \leq \lambda_{3} \leq \cdots
$$

where $\lambda_{1}$ is the smallest eigenvalue of $A_{g}$. In addition, there exist the corresponding collection of eigenfunctions $\left\{w_{i}\right\}_{i \in \mathbb{N}}$ forms an orthonormal basis for $H_{g}$.

Since the operators $A_{g}$ and $P_{g}$ are self-adjoint, using integration by parts we have

$$
\left\langle A_{g} u, u\right\rangle_{g}==\left\langle P_{g}\left[-\frac{1}{g}(\nabla \cdot g \nabla) u\right], u\right\rangle_{g}=\int_{\Omega}(\nabla u \cdot \nabla u) g d x=\langle\nabla u, \nabla u\rangle_{g} .
$$

Therefore,for $u \in V_{g}$ we can write $\left|A_{g}^{1 / 2} u\right|_{g}=|\nabla u|_{g}=\|u\|_{g}$.

Next, since the functional

$$
\tau \in W_{g} \rightarrow(\nabla \theta, \nabla \tau)_{g} \in \mathbb{R}
$$

is a continuous linear mapping on $W_{g}$, we can define a continuous linear mapping $\tilde{A}_{g}$ on $W_{g}^{\prime}$ such that

for all $\theta \in W_{g}$.

$$
\forall \tau \in W_{g}, \quad\left\langle\tilde{A}_{g} \theta, \tau\right\rangle_{g}=(\nabla \theta, \nabla \tau)_{g}
$$


We denote the bilinear operator $B_{g}(u, v)=P_{g}[(u \cdot \nabla) v]$ and the trilinear form

$$
b_{g}(u, v, w)=\sum_{i, j=1}^{n} \int_{\Omega} u_{i} \frac{\partial v_{j}}{\partial x_{i}} w_{j} g d x
$$

where $u, v, w$ lie in appropriate subspaces of $L_{p e r}^{2}(\Omega, g)$. Then, one obtains $b_{g}(u, v, w)=$ $-b_{g}(u, w, v)$ which implies that $b_{g}(u, v, v)=0$. Also $b_{g}$ satisfies the inequality (see [39],[42]),

$$
\left|b_{g}(u, v, w)\right|_{g} \leq c|u|_{g}^{1 / 2}\|u\|_{g}^{1 / 2}|v|_{g}|w|_{g}^{1 / 2}\|w\|_{g}^{1 / 2}, \forall u, v, w \in V_{g} .
$$

Similarly, for $u \in V_{g}$ and $\theta, \tau \in W_{g}$ we define $\tilde{B}_{g}(u, \theta)=\tilde{P}_{g}[(u \cdot \nabla) \theta]$ and

$$
\tilde{b}_{g}(u, \theta, \tau)=\sum_{i, j=1}^{n} \int_{\Omega} u_{i}(x) \frac{\partial \theta(x)}{\partial x_{j}} \tau(x) g d x .
$$

We denote the operators $C_{g} u=P_{g}\left[\frac{1}{g}(\nabla g \cdot \nabla) u\right]$ and $\tilde{C}_{g} \theta=\tilde{P}_{g}\left[\frac{1}{g}(\nabla g \cdot \nabla) \theta\right]$ such that

$$
\left\langle C_{g} u, v\right\rangle_{g}=b_{g}\left(\frac{\nabla g}{g}, u, v\right),\left\langle\tilde{C}_{g} \theta, \tau\right\rangle_{g}=\tilde{b}_{g}\left(\frac{\nabla g}{g}, \theta, \tau\right) .
$$

Finally, let $\tilde{D}_{g} \theta=\tilde{P}_{g}\left[\frac{\Delta g}{g} \theta\right]$ such that

$$
\left\langle\tilde{D}_{g} \theta, \tau\right\rangle_{g}=-\tilde{b}_{g}\left(\frac{\nabla g}{g}, \theta, \tau\right)-\tilde{b}_{g}\left(\frac{\nabla g}{g}, \tau, \theta\right) .
$$

We now rewrite the system of equations (2.4) - (2.6) as abstract evolutionary equations

$$
\begin{aligned}
\frac{d u}{d t}+B_{g}(u, u)+\nu A_{g} u+\nu C_{g} u & =\xi \theta+f_{1} \\
\frac{d \theta}{d t}+\tilde{B}_{g}(u, \theta)+\kappa \tilde{A}_{g} \theta-\kappa \tilde{C}_{g} \theta-\kappa \tilde{D}_{g} \theta & =f_{2} \\
u(x, 0)=u_{0}(x), \quad \theta(x, 0)=\theta_{0}(x) . &
\end{aligned}
$$

We give the following two lemmas and for the proofs of these lemmas we refer to $[27,1]$.

2.2. Lemma. For $n=2$ there exists a positive constant $c$ such that

$$
|u|_{L^{4}(\Omega, g)} \leq c|u|_{g}^{1 / 2}|\nabla u|_{g}^{1 / 2}, \forall u \in H^{1}(\Omega, g) .
$$

2.3. Lemma. For $u \in L^{2}\left(0, T ; V_{g}\right), B_{g}(u, u)(t) \in L^{1}\left(0, T ; V_{g}^{\prime}\right)$ and $C_{g} u(t) \in L^{2}\left(0, T ; H_{g}\right)$.

Lemma 2.2 is used in the proof of Lemma 2.3 and Lemma 2.3 is used when we are to show that the solution of the system is almost everywhere equal to a continuous function from $[0, T]$ to $V_{g}^{\prime}$ so that the solution satisfy the initial conditions.

\section{Existence and uniqueness of weak solutions}

3.1. Definition. A pair of functions $\{u, \theta\}$ is called a weak solution of $(2.4)-(2.6)$ if $u \in L^{2}\left(0, T ; V_{g}\right)$ and $\theta \in L^{2}\left(0, T ; W_{g}\right)$ satisfy the following equations

$$
\begin{array}{r}
\frac{d}{d t}(u, v)_{g}+b_{g}(u, u, v)+\nu(\nabla u, \nabla v)_{g}+\nu\left(C_{g} u, v\right)_{g}=(\xi \theta, v)_{g}+\left(f_{1}, v\right)_{g} \\
\frac{d}{d t}(\theta, \tau)_{g}+\tilde{b}_{g}(u, \theta, \tau)+\kappa(\nabla \theta, \nabla \tau)_{g}+\kappa \tilde{b}_{g}\left(\frac{\nabla g}{g}, \tau, \theta\right)=\left(f_{2}, v\right)_{g}
\end{array}
$$

for all $v \in V_{g}$ and $\tau \in W_{g}$.

3.2. Theorem. If $f_{1}, f_{2} \in L^{2}\left(0, T ; L^{2}(\Omega, g)\right), u_{0} \in H_{g}, \theta_{0} \in L^{2}(\Omega, g)$ and $g$ is a smooth function satisfying the conditions given in (2.1) defined on $\Omega_{2}$ then, there exist a unique weak solution $\{u, \theta\}$ of the system (2.8)-(2.10) satifying the periodic boundary conditions. 
For the proof of the theorem, we shall use the standard Feado - Galerkin method. Since $V_{g}$ is seperable and $V_{1}$ is dense in $V_{g}$, there exists a sequence $\left\{u_{i}\right\}_{i \in \mathbb{N}}$ which forms a complete orthonormal system in $H_{g}$ and a base for $V_{g}$. Similarly, there exists a sequence $\left\{\theta_{i}\right\}_{i \in \mathbb{N}}$ which forms a complete orthonormal system in $L^{2}(\Omega, g)$ and a base for $W_{g}$.

Let $\mathrm{m}$ be an arbitrary but fixed positive integer. For each $\mathrm{m}$ we define an approximate solution $\left\{u^{m}(t), \theta^{m}(t)\right\}$ of $(3.1)-(3.2)$ for $1 \leq k \leq m$ and $t \in[0, T]$ in the form,

$$
\begin{gathered}
u^{(m)}(t)=\sum_{j=1}^{m} f_{j}^{(m)}(t) u_{j}, \quad \theta^{(m)}(t)=\sum_{j=1}^{m} g_{j}^{(m)}(t) \theta_{j} \\
\frac{d}{d t}\left(u^{(m)}, u_{k}\right)_{g}+b_{g}\left(u^{(m)}, u^{(m)}, u_{k}\right)+\nu\left(\left(u^{(m)}, u_{k}\right)\right)_{g} \\
+\nu b_{g}\left(\frac{\nabla g}{g}, u^{(m)}, u_{k}\right)=\left(\xi \theta^{(m)}, u_{k}\right)_{g}+\left(f_{1}, u_{k}\right)_{g} \\
\frac{d}{d t}\left(\theta^{(m)}, \theta_{k}\right)_{g}+\tilde{b}_{g}\left(u^{(m)}, \theta^{(m)}, \theta_{k}\right)+\kappa\left(\left(\theta^{(m)}, \theta_{k}\right)\right)_{g} \\
+\kappa \tilde{b}_{g}\left(\frac{\nabla g}{g}, \theta_{k}, \theta^{(m)}\right)=\left(f_{2}, \theta_{k}\right)_{g} \\
u^{(m)}(0)=u_{m 0}=\sum_{j=1}^{m}\left(a_{0}, u_{j}\right) u_{j}, \quad \theta^{(m)}(0)=\theta_{m 0}=\sum_{j=1}^{m}\left(\tau_{0}, \theta_{j}\right) \theta_{j} .
\end{gathered}
$$

This system forms a nonlinear first order system of ordinary differential equations for the functions $f_{j}^{(m)}(t)$ and $g_{j}^{(m)}(t)$ and has a maximal solution on some interval [0,T](cf. [22]).

We multiply (3.4) and (3.5) by $f_{j}^{(m)}(t)$ and $g_{j}^{(m)}(t)$ respectively, and add these equations for $k=1, \ldots, m$. Taking into account $b_{g}\left(u^{(m)}, u^{(m)}, u^{(m)}\right)=0$ and $\tilde{b}_{g}\left(u^{(m)}, \theta^{(m)}, \theta^{(m)}\right)=$ 0 we get;

$$
\begin{array}{r}
\left(u^{\prime(m)}(t), u^{(m)}(t)\right)_{g}+\nu\left\|u^{(m)}(t)\right\|_{g}^{2}+\nu b_{g}\left(\frac{\nabla g}{g}, u^{(m)}(t), u^{(m)}(t)\right) \\
=\left(\xi \theta^{(m)}, u^{(m)}(t)\right)_{g}+\left(f_{1}, u^{(m)}(t)\right) \\
\left(\theta^{\prime(m)}(t), \theta^{(m)}(t)\right)_{g}+\kappa\left\|\theta^{(m)}(t)\right\|_{g}^{2}+\kappa \tilde{b}_{g}\left(\frac{\nabla g}{g}, \theta^{(m)}(t), \theta^{(m)}(t)\right) \\
=\left(f_{2}, \theta^{(m)}(t)\right)_{g} .
\end{array}
$$

Using Schwarz and Young inequalities in (3.7) and (3.8)

$$
\begin{aligned}
\frac{d}{d t}\left|u^{(m)}(t)\right|_{g}^{2}+\nu\left\|u^{(m)}(t)\right\|_{g}^{2} & \leq \frac{M_{0}|\xi|_{\infty}^{2}}{\pi^{2} m_{0} \nu}\left|\theta^{(m)}(t)\right|_{g}^{2}+\frac{4}{\nu}\left\|f_{1}(t)\right\|_{V_{g}^{\prime}}^{2}+\frac{2 \nu|\nabla g|_{\infty}^{2}}{m_{0}^{2}}\left|u^{(m)}(t)\right|_{g}^{2} \\
\frac{d}{d t}\left|\theta^{(m)}(t)\right|_{g}^{2}+\kappa\left\|\theta^{(m)}(t)\right\|_{g}^{2} & \leq \frac{2}{\kappa}\left\|f_{2}(t)\right\|_{W_{g}^{\prime}}^{2}+\frac{2 \kappa|\nabla g|_{\infty}^{2}}{m_{0}^{2}}\left|\theta^{(m)}(t)\right|_{g}^{2}
\end{aligned}
$$

so that for $|\nabla g|_{\infty}^{2}<\frac{\pi^{2} m_{0}^{3}}{M_{0}}$ and for

$$
\nu^{\prime}=\nu\left(1-\frac{M_{0}|\nabla g|_{\infty}^{2}}{2 \pi^{2} m_{0}^{3}}\right), \quad \kappa^{\prime}=\kappa\left(1-\frac{M_{0}|\nabla g|_{\infty}^{2}}{2 \pi^{2} m_{0}^{3}}\right), \quad c^{\prime}=\frac{M_{0}^{2}\|\xi\|_{\infty}^{2}}{4 \pi^{4} m_{0}^{2}}
$$

we get the inequalities

$$
\begin{aligned}
\frac{d}{d t}\left|u^{(m)}(t)\right|_{g}^{2}+\nu^{\prime}\left\|u^{(m)}(t)\right\|_{g}^{2} & \leq \frac{c^{\prime}}{\nu}\left\|\theta^{(m)}(t)\right\|_{g}^{2}+\frac{4}{\nu}\left\|f_{1}\right\|_{V_{g}^{\prime}}^{2} \\
\frac{d}{d t}\left|\theta^{(m)}(t)\right|_{g}^{2}+\kappa^{\prime}\left\|\theta^{(m)}(t)\right\|_{g}^{2} & \leq \frac{2}{\kappa}\left\|f_{2}\right\|_{W_{g}^{\prime}}^{2} .
\end{aligned}
$$


Integrating (3.9) and (3.10) from 0 to $t$ we obtain

$$
\begin{array}{r}
\sup _{t \in[0, T]}\left|u^{(m)}(t)\right|_{g}^{2} \leq\left|u_{0}\right|_{g}^{2}+\frac{c^{\prime}}{\nu \kappa^{\prime}}\left|\theta_{0}\right|_{g}^{2}+\frac{4}{\nu} \int_{0}^{T}\left\|f_{1}(s)\right\|_{V_{g}^{\prime}}^{2} d s \\
+\frac{2 c^{\prime}}{\nu \kappa \kappa^{\prime}} \int_{0}^{T}\left\|f_{2}(s)\right\|_{W_{g}^{\prime}}^{2} d s \\
\sup _{t \in[0, T]}\left|\theta^{(m)}(t)\right|_{g}^{2} \leq\left|\theta_{0}\right|_{g}^{2}+\frac{2}{\kappa} \int_{0}^{T}\left\|f_{2}(s)\right\|_{W_{g}^{\prime}}^{2} d s
\end{array}
$$

which imply that the sequences $\left\{u^{(m)}\right\}_{m}$ and $\left\{\theta^{(m)}\right\}_{m}$ remain in a bounded set of $L^{\infty}\left(0, T ; H_{g}\right)$ and $L^{\infty}\left(0, T ; L^{2}(\Omega, g)\right)$ respectively.

We then integrate (3.9) and (3.10) from 0 to $T$ to get

$$
\begin{aligned}
\left|u^{(m)}(T)\right|_{g}^{2}+\nu^{\prime} \int_{0}^{T}\left\|u^{(m)}(t)\right\|_{g}^{2} d t \leq & \frac{2 c^{\prime}}{\nu \kappa \kappa^{\prime}} \int_{0}^{T}\left\|f_{2}(t)\right\|_{W_{g}^{\prime}}^{2} d t \\
& +\frac{4}{\nu} \int_{0}^{T}\left\|f_{1}(t)\right\|_{V_{g}^{\prime}}^{2} d t \\
\left|\theta^{(m)}(T)\right|_{g}^{2}+\kappa^{\prime} \int_{0}^{T}\left\|\theta^{(m)}(t)\right\|_{g}^{2} d t & \leq \frac{2}{\kappa} \int_{0}^{T}\left\|f_{2}(t)\right\|_{W_{g}^{\prime}}^{2} d t
\end{aligned}
$$

which imply that the sequences $\left\{u^{(m)}\right\}_{m}$ and $\left\{\theta^{(m)}\right\}_{m}$ remain in a bounded set of $L^{2}\left(0, T ; V_{g}\right)$ and $L^{2}\left(0, T ; W_{g}\right)$ respectively. Due to the estimates (3.11)-(3.14) we assert the existence of elements $u \in L^{2}\left(0, T ; V_{g}\right) \cap L^{\infty}\left(0, T ; H_{g}\right)$ and $\theta \in L^{2}\left(0, T ; W_{g}\right) \cap$ $L^{\infty}\left(0, T ; L^{2}(\Omega, g)\right)$ and the subsequences $\left\{u^{(m)}\right\}_{m}$ and $\left\{\theta^{(m)}\right\}_{m}$ such that $u^{(m)} \rightarrow u \in$ $L^{2}\left(0, T ; V_{g}\right)$ and $\theta^{(m)} \rightarrow \theta \in L^{2}\left(0, T ; W_{g}\right)$ weakly and $u^{(m)} \rightarrow u \in L^{\infty}\left(0, T ; H_{g}\right)$ and $\theta^{(m)} \rightarrow \theta \in L^{\infty}\left(0, T ; L^{2}(\Omega, g)\right)$ weak-star convergent as $m \rightarrow \infty$.

Let $\tilde{u}^{(m)}: \mathbb{R} \rightarrow V_{g}$ and $\tilde{\theta}^{(m)}: \mathbb{R} \rightarrow W_{g}$ defined as

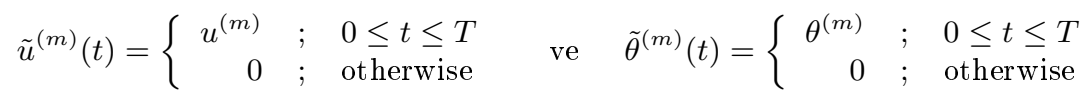

and their Fourier transforms denoted by $\hat{u}^{(m)}$ ve $\hat{\theta}^{(m)}$.

We observe that

$$
\begin{aligned}
& \frac{d}{d t} \tilde{u}^{(m)}={\tilde{u^{\prime}}}^{(m)}+u^{(m)}(0) \delta_{0}-u^{(m)}(T) \delta_{T} \\
& \frac{d}{d t} \tilde{\theta}^{(m)}={\tilde{\theta^{\prime}}}^{(m)}+\theta^{(m)}(0) \delta_{0}-\theta^{(m)}(T) \delta_{T}
\end{aligned}
$$

where $\delta_{0}$ and $\delta_{T}$ are Dirac distributions at 0 and $T$. Therefore, by Fourier transform we get

$$
\begin{aligned}
2 i \pi \tau\left\langle\hat{u}^{(m)}, u_{k}\right\rangle_{g} & =\left\langle\hat{F}_{1}, u_{k}\right\rangle_{g}+\left(u_{m 0}, u_{k}\right)_{g}-\left(u^{(m)}(T), u_{k}\right)_{g} e^{-2 i \pi T \tau} \\
2 i \pi \tau\left\langle\hat{\theta}^{(m)}, \theta_{k}\right\rangle_{g} & =\left\langle\hat{F}_{2}, \theta_{k}\right\rangle_{g}+\left(\theta_{m 0}, \theta_{k}\right)_{g}-\left(\theta^{(m)}(T), \theta_{k}\right)_{g} e^{-2 i \pi T \tau}
\end{aligned}
$$

where

$$
\begin{aligned}
& F_{1}=\xi \theta^{(m)}+f_{1}-B_{g}\left(u^{(m)}, u^{(m)}\right)-\nu A_{g} u^{(m)}-\nu C_{g} u^{(m)} \\
& F_{2}=f_{2}-\tilde{B}_{g}\left(u^{(m)}, \theta^{(m)}\right)-\kappa \tilde{A}_{g} \theta^{(m)}+\kappa \tilde{C}_{g} \theta^{(m)}+\kappa \tilde{D}_{g} \theta^{(m)}
\end{aligned}
$$

for $k=1, \ldots, m$. We multiply $(3.15)$ and $(3.16)$ by $\hat{f}_{j}^{(m)}$ ve $\hat{g}_{j}^{(m)}$ respectively and add these equations for $k=1, \ldots, m$ to get

$$
\begin{aligned}
2 i \pi \tau\left|\hat{u}^{(m)}(\tau)\right|_{g}^{2} & =\left\langle\hat{F}_{1}(\tau), \hat{u}^{(m)}(\tau)\right\rangle_{g}+\left(u_{m 0}, \hat{u}^{(m)}(\tau)\right)_{g}-\left(u^{(m)}(T), \hat{u}^{(m)}(\tau)\right)_{g} e^{-2 i \pi T \tau} \\
2 i \pi \tau\left|\hat{\theta}^{(m)}(\tau)\right|_{g}^{2} & =\left\langle\hat{F}_{2}, \hat{\theta}^{(m)}(\tau)\right\rangle_{g}+\left(\theta_{m 0}, \hat{\theta}^{(m)}(\tau)\right)_{g}-\left(\theta^{(m)}(T), \hat{\theta}^{(m)} \cdot(\tau)\right)_{g} e^{-2 i \pi T \tau}
\end{aligned}
$$


Since the integrals on the right hand side of the inequalities

$$
\begin{array}{r}
\int_{0}^{T}\left\|F_{1}(t)\right\|_{V_{g}^{\prime}} d t \leq \int_{0}^{T} c\left(|\xi|_{\infty}\left\|\theta^{(m)}(t)\right\|_{g}+\left\|f_{1}(t)\right\|_{V_{g}^{\prime}}+\left|u^{(m)}(t)\right|_{g}\left\|u^{(m)}\right\|_{g}+\right. \\
\left.\left\|u^{(m)}(t)\right\|_{g}+|\nabla g|_{\infty}\left\|u^{(m)}(t)\right\|_{g}\right) d t \\
\int_{0}^{T}\left\|F_{2}(t)\right\|_{W_{g}^{\prime}} d t \leq \int_{0}^{T} \grave{c}\left(\left\|f_{2}(t)\right\|_{W_{g}^{\prime}}+\left|u^{(m)}(T)\right|_{g}\left\|\theta^{(m)}(t)\right\|_{g}+\left\|\theta^{(m)}(t)\right\|_{g}\right. \\
\left.+|\nabla g|_{\infty}\left\|\theta^{(m)}(t)\right\|_{g}+|\Delta g|_{\infty}\left\|\theta^{(m)}(t)\right\|_{g}\right) d t
\end{array}
$$

remains bounded, $\left\|F_{1}(t)\right\|_{V_{g}^{\prime}}$ and $\left\|F_{2}(t)\right\|_{W_{g}^{\prime}}$ are bounded in $L^{1}\left(0, T ; V_{g}^{\prime}\right)$ and $L^{1}\left(0, T ; W_{g}^{\prime}\right)$ respectively. Therefore, $\forall m$

$$
\sup _{\tau \in \mathbb{R}}\left\|\hat{F}_{1}(\tau)\right\|_{V_{g}^{\prime}} \leq c_{1} \quad \text { and } \quad \sup _{\tau \in \mathbb{R}}\left\|\hat{F}_{2}(\tau)\right\|_{W_{g}^{\prime}} \leq c_{2}
$$

Moreover, since $u^{(m)}(0), u^{(m)}(T), \theta^{(m)}(0)$ and $\theta^{(m)}(T)$ are bounded by the inequalities (3.11) and (3.12),

$$
\begin{aligned}
|\tau|\left|\tilde{u}^{(m)}(\tau)\right|_{g}^{2} & \leq c_{1}\left\|u^{(m)}\right\|_{V_{g}}+c_{2}\left|u^{(m)}\right|_{g} \leq c_{3}\left\|u^{(m)}\right\|_{V_{g}} \\
|\tau|\left|\tilde{\theta}^{(m)}(\tau)\right|_{g}^{2} & \leq \grave{c_{1}}\left\|\theta^{(m)}\right\|_{W_{g}}+\grave{c_{2}}\left|\theta^{(m)}\right|_{g} \leq \grave{c_{3}}\left\|\theta^{(m)}\right\|_{W_{g}}
\end{aligned}
$$

and for $\gamma<\frac{1}{4}$ fixed we observe that

$$
|\tau|^{2 \gamma} \leq c(\gamma) \frac{1+|\tau|}{1+|\tau|^{1-2 \gamma}}, \quad \forall \tau \in \mathbb{R}
$$

then we can write

$$
\begin{aligned}
\int_{-\infty}^{\infty}|\tau|^{2 \gamma}\left|\hat{u}^{(m)}(\tau)\right|_{g}^{2} d \tau & \leq c(\gamma) \int_{-\infty}^{\infty} \frac{1+|\tau|}{1+|\tau|^{1-2 \gamma}}\left|\hat{u}^{(m)}(\tau)\right|_{g}^{2} d \tau \\
& \leq c_{4} \int_{-\infty}^{\infty} \frac{1}{1+|\tau|^{1-2 \gamma}}\left\|\hat{u}^{(m)}(\tau)\right\|_{V_{g}}^{2} d \tau+c_{5} \int_{-\infty}^{\infty}\left\|\hat{u}^{(m)}(\tau)\right\|_{V_{g}}^{2} d \tau \\
\int_{-\infty}^{\infty}|\tau|^{2 \gamma}\left|\hat{\theta}^{(m)}(\tau)\right|_{g}^{2} d \tau & \leq \grave{c_{4}} \int_{-\infty}^{\infty} \frac{1}{1+|\tau|^{1-2 \gamma}}\left\|\hat{\theta}^{(m)}(\tau)\right\|_{W_{g}}^{2} d \tau+\grave{c}_{5} \int_{-\infty}^{\infty}\left\|\hat{\theta}^{(m)}(\tau)\right\|_{W_{g}}^{2} d \tau .
\end{aligned}
$$

Since the integrals on the right hand side are bounded we see that $u^{(m)} \in H^{\gamma}\left(\mathbb{R} ; V_{g}, H_{g}\right)$ and $\theta^{(m)} \in H^{\gamma}\left(\mathbb{R} ; W_{g}, L^{2}(\Omega, g)\right)$. For the definitions of $H^{\gamma}\left(\mathbb{R} ; V_{g}, H_{g}\right)$ and $H^{\gamma}\left(\mathbb{R} ; W_{g}, L^{2}(\Omega, g)\right)$ we refer to [39]. Therefore, there exist subsequences $\left\{u^{(m)}\right\}_{m}$ and $\left\{\theta^{(m)}\right\}_{m}$ which are strongly convergent in $L^{2}\left(0, T ; H_{g}\right)$ and $L^{2}\left(0, T ; L^{2}(\Omega, g)\right)$ respectively.

In order to pass to the limit, we consider the scalar functions $\Psi_{1}(t)$ and $\Psi_{2}(t)$ continuously differentiable on $[0, T]$ and such that $\Psi_{1}(T)=0$ ve $\Psi_{2}(T)=0$. We multiply (3.4) and (3.5) by $\Psi_{1}(t)$ ve $\Psi_{2}(t)$ respectively and then integrate by parts.

$$
\begin{array}{r}
-\int_{0}^{T}\left(u^{(m)}, \Psi_{1}^{\prime} u_{k}\right)_{g} d t+\int_{0}^{T} b_{g}\left(u^{(m)}, u^{(m)}, \Psi_{1} u_{k}\right) d t+\nu \int_{0}^{T}\left(\left(u^{(m)}, \Psi_{1} u_{k}\right)\right)_{g} d t \\
+\nu \int_{0}^{T} b_{g}\left(\frac{\nabla g}{g}, u^{(m)}, \Psi_{1} u_{k}\right) d t=\left(u_{m 0}, u_{k}\right)_{g} \Psi_{1}(0)+\int_{0}^{T}\left(\xi \theta^{(m)}, \Psi_{1} u_{k}\right)_{g} d t+\int_{0}^{T}\left(f_{1}, u_{k}\right)_{g} d t, \\
-\int_{0}^{T}\left(\theta^{(m)}, \Psi_{2}^{\prime} \theta_{k}\right)_{g} d t+\int_{0}^{T} \tilde{b}_{g}\left(u^{(m)}, \theta^{(m)}, \Psi_{2} \theta_{k}\right) d t+\kappa \int_{0}^{T}\left(\left(\theta^{(m)}, \Psi_{2} \theta_{k}\right)\right)_{g} d t \\
+\kappa \int_{0}^{T} \tilde{b}_{g}\left(\frac{\nabla g}{g}, \theta_{k}, \Psi_{2} \theta^{(m)}\right) d t=\left(\theta_{m 0}, \theta_{k}\right)_{g} \Psi_{2}(0)+\int_{0}^{T}\left(f_{2}, \Psi_{2} \theta_{k}\right)_{g} d t .
\end{array}
$$


Following the technique given in [39, 3], as $m \rightarrow \infty$ we obtain

$$
\begin{array}{r}
-\int_{0}^{T}\left(u, \Psi_{1}^{\prime} v\right)_{g} d t+\int_{0}^{T} b_{g}\left(u, u, \Psi_{1} v\right) d t+\nu \int_{0}^{T}\left(\left(u, \Psi_{1} v\right)\right)_{g} d t \\
+\nu \int_{0}^{T} b_{g}\left(\frac{1}{g} \nabla g, u, \Psi_{1} v\right) d t=\left(u_{0}, v\right)_{g} \Psi_{1}(0)+\int_{0}^{T}\left(\xi \theta, \Psi_{1} v\right)_{g} d t+\int_{0}^{T}\left(f_{1}, v\right)_{g} d t \\
-\int_{0}^{T}\left(\theta, \Psi_{2}^{\prime} \tau\right)_{g} d t+\int_{0}^{T} \tilde{b}_{g}\left(u, \theta, \Psi_{2} \tau\right) d t+\kappa \int_{0}^{T}\left(\left(\theta, \Psi_{2} \tau\right)\right)_{g} d t \\
+\kappa \int_{0}^{T} \tilde{b}_{g}\left(\frac{\nabla g}{g}, \tau, \Psi_{2} \theta\right) d t=\left(\theta_{0}, \tau\right)_{g} \Psi_{2}(0)+\int_{0}^{T}\left(f_{2}, \Psi_{2} \tau\right)_{g} d t
\end{array}
$$

The equations (3.17) and (3.18) hold for $v$ and $\tau$ which are finite linear combinations of the $u_{k}$ and $\theta_{k}$ for $k=1, \ldots, m$ and by continuity (3.17) and (3.18) hold for $v \in V_{g}$ and $\tau \in H_{g}$ respectively. Rewriting (3.17) and (3.18) for $\Psi_{1}(t), \Psi_{2}(t) \in C_{0}^{\infty}(0, T)$ we see that $\{u, \theta\}$ satisfy (3.1) and (3.2). Furthermore, applying similar techniques given in [39, 1] it is easy to show that $\{u, \theta\}$ satisfy the initial conditions $u(0)=u_{0}$ and $\theta(0)=\theta_{0}$.

For the uniqueness of the weak solutions let $\left(u_{1}, \theta_{1}\right)$ and $\left(u_{2}, \theta_{2}\right)$ be two weak solutions with the same initial condition. Let $w=u_{1}-u_{2}$ and $\tilde{w}=\theta_{1}-\theta_{2}$. Then we have

$$
\begin{array}{r}
\frac{d}{d t}(w, v)_{g}+b_{g}\left(u_{1}, u_{1}, v\right)-b_{g}\left(u_{2}, u_{2}, v\right)+\nu(\nabla w, \nabla v)_{g}+\nu\left(C_{g} w, v\right)_{g}=(\xi \tilde{w}, v)_{g} \\
\frac{d}{d t}(\tilde{w}, \tau)_{g}+\tilde{b}_{g}\left(u_{1}, \theta_{1}, \tau\right)-\tilde{b}_{g}\left(u_{2}, \theta_{2}, \tau\right)+\kappa(\nabla \tilde{w}, \nabla \tau)_{g}+\kappa \tilde{b}_{g}\left(\frac{\nabla g}{g}, \tau, \tilde{w}\right)=0 .
\end{array}
$$

Taking $v=w(t)$ and $\tau=\tilde{w}(t)$ one obtains,

$$
\begin{array}{r}
\frac{1}{2} \frac{d}{d t}|w|_{g}^{2}+b_{g}\left(w, u_{2}, w\right)+\nu\left|A_{g}^{1 / 2} w\right|_{g}^{2}+\nu\left(C_{g} w, w\right)_{g}=(\xi \tilde{w}, w)_{g} \\
\frac{1}{2} \frac{d}{d t}|\tilde{w}|_{g}^{2}+\tilde{b}_{g}\left(u_{1}, \theta_{1}, \tilde{w}\right)-\tilde{b}_{g}\left(u_{2}, \theta_{2}, \tilde{w}\right)+\kappa\left|\tilde{A}_{g}^{1 / 2} \tilde{w}\right|_{g}^{2}+\kappa \tilde{b}_{g}\left(\frac{\nabla g}{g}, \tilde{w}, \tilde{w}\right)=0 .
\end{array}
$$

By applying the bounds on the terms $b_{g}, \tilde{b}_{g}$ it then follows by Cauchy-Schwarz inequality and Gronwall inequality that $w(t)=0$ and $\tilde{w}(t)=0$ for all $t \geq 0$ since we have $w(0)=0$ and $\tilde{w}(0)=0$. Thus Theorem 3.2 is proved.

\section{Determining modes}

In the 1960 s the theory of determining modes was introduced by Foias and Prodi [8]. Jones and Titi, presented improved upper bounds on the number of determining Fourier modes, determining nodes and volume elements for the Navier - Stokes equations in [20]. The dependence of the number of numerically determining modes in the Navier - Stokes equations on the Grashof number is examined in [32]. In [31] the connection between continuous data assimilation and the theory of determining modes was made clear. Foias et.al [5], presented a unified approach based on interpolant operators constructed from various determining parameters for the Navier-Stokes equations. Boland and Layton[2], analyze the error in finite element methods in approximating natural convection problems. They show that the steady state Boussinesq equations have at least one solution and it is unique for either small data or for restricted Rayleigh and Prandtl numbers. Also, they give an abstract formulation of a determining modes result which gives more structure to the uniqueness problem for large Rayleigh and Prandtl values. Quite recently, an algorithm for continuous data assimilation for the two dimensional Bénard convection problem is introduced and analyzed in [4].

Following [6] we first give a generalization of the classical Gronwall lemma. 
4.1. Lemma. [19] Let $\alpha=\alpha(t)$ and $\beta=\beta(t)$ be locally integrable real-valued functions on $[0, \infty)$ that satisfy the following conditions for some $T>0$ :

$$
\begin{array}{r}
\liminf _{t \rightarrow \infty} \frac{1}{T} \int_{t}^{t+T} \alpha(\tau) d \tau>0 \\
\limsup _{t \rightarrow \infty} \frac{1}{T} \int_{t}^{t+T} \alpha^{-}(\tau) d \tau<\infty \\
\lim _{t \rightarrow \infty} \frac{1}{T} \int_{t}^{t+T} \beta^{+}(\tau) d \tau=0
\end{array}
$$

where $\alpha^{-}(t)=\max \{-\alpha(t), 0\}$ and $\beta^{+}(t)=\max \{\beta(t), 0\}$. Suppose that $\xi=\xi(t)$ is an absolutely continuous nonnegative function on $[0, \infty)$ that satisfies the following inequality almost everywhere $[0, \infty)$ :

$$
\frac{d \xi}{d t}+\alpha \xi \leq \beta
$$

Then $\xi(t) \rightarrow 0$ as $t \rightarrow \infty$.

Now with the use of Lemma 4.1 we give a proof for upper bounds for the determining modes to the system of equations under consideration.

Let $(u, \theta)$ and $(\bar{u}, \bar{\theta})$ satisy

$$
\begin{array}{r}
u_{t}+\nu A_{g} u+B_{g}(u, u)+\nu C_{g} u=\xi \theta+f_{1} \\
\theta_{t}+\kappa \tilde{A}_{g} \theta+\tilde{B}_{g}(u, \theta)-\kappa \tilde{C}_{g} \theta-\kappa \tilde{D}_{g} \theta=f_{2} \\
\bar{u}_{t}+\nu A_{g} \bar{u}+B_{g}(\bar{u}, \bar{u})+\nu C_{g} \bar{u}=\xi \bar{\theta}+\bar{f}_{1} \\
\bar{\theta}_{t}+\kappa \tilde{A}_{g} \bar{\theta}+\tilde{B}_{g}(\bar{u}, \bar{\theta})-\kappa \tilde{C}_{g} \bar{\theta}-\kappa \tilde{D}_{g} \bar{\theta}=\bar{f}_{2}
\end{array}
$$

the system of equations (4.5-4.6) and (4.7-4.8) respectively under periodic boundary conditions and corresponding to two possibly different forcing pairs $\left\{f_{1}=f_{1}(x, t), \bar{f}_{1}=\right.$ $\left.\bar{f}_{1}(x, t)\right\}$ and $\left\{f_{2}=f_{2}(x, t), \bar{f}_{2}=\bar{f}_{2}(x, t)\right\}$. We can expand each solution pair in the form with the Galerkin projections correspond to the first $m$ modes:

$$
\begin{array}{r}
u=\sum_{j=1}^{\infty} \breve{u}_{j}(t) u_{j}(x), \quad \theta=\sum_{j=1}^{\infty} \breve{\theta}_{j}(t) \theta_{j}(x) \\
P_{m} u=\sum_{j=1}^{m} \breve{u}_{j}(t) u_{j}(x), \quad \tilde{P}_{m} \theta=\sum_{j=1}^{m} \breve{\theta}_{j}(t) \theta_{j}(x)
\end{array}
$$

where $\breve{u}_{j}$ and $\breve{\theta}_{j}$ are eigenfunctions of the g-Stokes operator. It is assumed that the forcing pairs $\left\{f_{1}, \bar{f}_{1}\right\}$ and $\left\{f_{2}, \bar{f}_{2}\right\}$ have the same asymptotic behavior for large time, that is, as $t \rightarrow \infty$ we have

$$
\int_{\Omega}\left|f_{1}-\bar{f}_{1}\right|_{V_{g}^{\prime}}^{2} d x \rightarrow 0, \quad \int_{\Omega}\left|f_{2}-\bar{f}_{2}\right|_{W_{g}^{\prime}}^{2} d x \rightarrow 0 .
$$

Then, the first $m$ modes associated with $P_{m}$ and $\tilde{P}_{m}$ are called determining modes if the condition

$$
\int_{\Omega}\left|P_{m} u-P_{m} \bar{u}\right|_{g}^{2} d x \rightarrow 0, \quad \int_{\Omega}\left|\tilde{P}_{m} \theta-\tilde{P}_{m} \bar{\theta}\right|_{g}^{2} d x \rightarrow 0
$$

imply

$$
\int_{\Omega}|u-\bar{u}|_{g}^{2} d x \rightarrow 0, \quad \int_{\Omega}|\theta-\bar{\theta}|_{g}^{2} d x \rightarrow 0
$$


as $t \rightarrow \infty$. By subtraction, we find that

$$
\begin{array}{r}
\omega_{t}+\nu A_{g} \omega+B_{g}(u, u)-B_{g}(\bar{u}, \bar{u})+\nu C_{g} \omega=\xi \tilde{\omega}+f_{1}-\bar{f}_{1} \\
\tilde{\omega}_{t}+\kappa \tilde{A}_{g} \tilde{\omega}+\tilde{B}_{g}(u, \theta)-\tilde{B}_{g}(\bar{u}, \bar{\theta})-\kappa \tilde{C}_{g} \tilde{\omega}-\kappa \tilde{D}_{g} \tilde{\omega}=f_{2}-\bar{f}_{2}
\end{array}
$$

where $\omega=u-\bar{u}$ and $\tilde{\omega}=\theta-\bar{\theta}$. By using

$$
\begin{aligned}
B_{g}(u, u)-B_{g}(\bar{u}, \bar{u}) & =B_{g}(\omega, u)+B_{g}(\bar{u}, \omega) \\
\tilde{B}_{g}(u, \theta)-\tilde{B}_{g}(\bar{u}, \bar{\theta}) & =\frac{1}{2}\left[\tilde{B}_{g}(\omega, \theta)+\tilde{B}_{g}(\omega, \bar{\theta})+\tilde{B}_{g}(u, \tilde{\omega})+\tilde{B}_{g}(\bar{u}, \tilde{\omega})\right]
\end{aligned}
$$

and taking the inner product of (4.12) and (4.13) with $Q_{m}=I-P_{m}$ and $\tilde{Q}_{m}=I-\tilde{P}_{m}$ which are the projections onto the modes higher than $m$ respectively, yields

$$
\begin{array}{r}
\left(\omega_{t}, Q_{m} \omega\right)_{g}+\nu\left(A_{g} \omega, Q_{m} \omega\right)_{g}+b_{g}\left(\omega, u, Q_{m} \omega\right)+b_{g}\left(\bar{u}, \omega, Q_{m} \omega\right)+\nu b_{g}\left(\frac{\nabla g}{g}, \omega, Q_{m} \omega\right) \\
=\left(\xi \tilde{\omega}, Q_{m} \omega\right)_{g}+\left(f_{1}-\bar{f}_{1}, Q_{m} \omega\right)_{g} \\
\left(\tilde{\omega}_{t}, \tilde{Q}_{m} \tilde{\omega}\right)_{g}+\kappa\left(\tilde{A}_{g} \tilde{\omega}, \tilde{Q}_{m} \tilde{\omega}\right)_{g}+\frac{1}{2}\left[\tilde{b}_{g}\left(\omega, \theta, \tilde{Q}_{m} \tilde{\omega}\right)+\tilde{b}_{g}\left(\omega, \bar{\theta}, \tilde{Q}_{m} \tilde{\omega}\right)+\tilde{b}_{g}\left(u, \tilde{\omega}, \tilde{Q}_{m} \tilde{\omega}\right)\right. \\
\left.+\tilde{b}_{g}\left(\bar{u}, \tilde{\omega}, \tilde{Q}_{m} \tilde{\omega}\right)\right]+\kappa \tilde{b}_{g}\left(\frac{\nabla g}{g}, \tilde{Q}_{m} \tilde{\omega}, \tilde{\omega}\right)=\left(f_{2}-\bar{f}_{2}, \tilde{Q}_{m} \tilde{\omega}\right)_{g} .
\end{array}
$$

Therefore,

$$
\begin{array}{r}
\frac{1}{2} \frac{d}{d t}\left|Q_{m} \omega\right|_{g}^{2}+\nu\left\|Q_{m} \omega\right\|_{g}^{2}+b_{g}\left(\omega, u, Q_{m} \omega\right)+b_{g}\left(\bar{u}, \omega, Q_{m} \omega\right) \\
+\nu b_{g}\left(\frac{\nabla g}{g}, \omega, Q_{m} \omega\right)=\left(\xi \tilde{\omega}, Q_{m} \omega\right)_{g}+\left(f_{1}-\bar{f}_{1}, Q_{m} \omega\right)_{g} \\
\frac{1}{2} \frac{d}{d t}\left|\tilde{Q}_{m} \tilde{\omega}\right|_{g}^{2}+\kappa\left\|\tilde{Q}_{m} \tilde{\omega}\right\|_{g}^{2}+\frac{1}{2}\left[\tilde{b}_{g}\left(\omega, \theta, \tilde{Q}_{m} \tilde{\omega}\right)+\tilde{b}_{g}\left(\omega, \bar{\theta}, \tilde{Q}_{m} \tilde{\omega}\right)\right. \\
\left.+\tilde{b}_{g}\left(u, \tilde{\omega}, \tilde{Q}_{m} \tilde{\omega}\right)+\tilde{b}_{g}\left(\bar{u}, \tilde{\omega}, \tilde{Q}_{m} \tilde{\omega}\right)\right]+\kappa \tilde{b}_{g}\left(\frac{\nabla g}{g}, \tilde{Q}_{m} \tilde{\omega}, \tilde{\omega}\right)=\left(f_{2}-\bar{f}_{2}, \tilde{Q}_{m} \tilde{\omega}\right)_{g} .
\end{array}
$$

By using the estimate (2.7), Cauchy - Schwarz inequality and Young's inequality we give some bounds on the terms which occur in the equations (4.14) and (4.15).

$$
\begin{aligned}
b_{g}\left(\omega, u, Q_{m} \omega\right) & =b_{g}\left(P_{m} \omega, u, Q_{m} \omega\right)+b_{g}\left(Q_{m} \omega, u, Q_{m} \omega\right) \\
\leq & c_{1}\left|P_{m} \omega\right|_{g}^{1 / 2}\left\|P_{m} \omega\right\|_{g}^{1 / 2}\|u\|_{g}\left|Q_{m} \omega\right|_{g}^{1 / 2}\left\|Q_{m} \omega\right\|_{g}^{1 / 2}+\frac{5 c_{2}^{2}}{2 \nu}\left|Q_{m} \omega\right|_{g}^{2}\|u\|_{g}^{2} \\
& +\frac{\nu}{10}\left\|Q_{m} \omega\right\|_{g}^{2} \\
\nu b_{g}\left(\frac{\nabla g}{g}, \omega, Q_{m} \omega\right) \leq & c_{1} \nu|\nabla g|_{\infty}\left\|P_{m} \omega\right\|_{g}\left|Q_{m} \omega\right|_{g}+\frac{5 c_{2}^{2} \nu}{2}|\nabla g|_{\infty}^{2}\left|Q_{m} \omega\right|_{g}^{2}+\frac{\nu}{10}\left\|Q_{m} \omega\right\|_{g}^{2} \\
\frac{1}{2} \tilde{b}_{g}\left(\omega, \theta, \tilde{Q}_{m} \tilde{\omega}\right) & \leq \frac{c_{1}}{2}\left|P_{m} \omega\right|_{g}^{1 / 2}\left\|P_{m} \omega\right\|_{g}^{1 / 2}|\theta|_{g}^{1 / 2}\|\theta\|_{g}^{1 / 2}\left\|\tilde{Q}_{m} \tilde{\omega}\right\|_{g}+\frac{c_{2}^{2}}{2}\left|Q_{m} \omega\right|_{g}\left\|Q_{m} \omega\right\|_{g} \\
& +\frac{1}{8}\|\theta\|_{g}^{2}\left|\tilde{Q} \tilde{Q}_{m} \tilde{\omega}\right|_{g}\left\|\tilde{Q} \tilde{Q}_{m} \tilde{\omega}\right\|_{g} \\
& \leq \frac{c_{1}}{2}\left|P_{m} \omega\right|_{g}^{1 / 2}\left\|P_{m} \omega\right\|_{g}^{1 / 2}|\theta|_{g}^{1 / 2}\|\theta\|_{g}^{1 / 2}\left\|\tilde{Q} \tilde{\omega}_{m} \tilde{\omega}\right\|_{g} \\
& +\frac{5 c_{2}^{4}}{8 \nu}\left|Q_{m} \omega\right|_{g}^{2}+\frac{\nu}{10}\left\|Q_{m} \omega\right\|_{g}^{2}+\frac{3}{128 \kappa}\|\theta\|_{g}^{4}\left|\tilde{Q_{m}} \tilde{\omega}\right|_{g}^{2}+\frac{\kappa}{6}\left\|\tilde{Q} \tilde{Q}_{m} \tilde{\omega}\right\|_{g}^{2}
\end{aligned}
$$


Similarly we obtain bounds for the rest of the terms in the equations (4.14) and (4.15) then taking these estimates into account and using the inequalities

$$
\lambda_{m+1}\left|Q_{m} \omega\right|_{g}^{2} \leq\left\|Q_{m} \omega\right\|_{g}^{2}, \quad \lambda_{m+1}\left|\tilde{Q}_{m} \tilde{\omega}\right|_{g}^{2} \leq\left\|\tilde{Q}_{m} \tilde{\omega}\right\|_{g}^{2}
$$

we obtain a relation for $\zeta(t)=\left|Q_{m} \omega\right|^{2}+\left|\tilde{Q}_{m} \tilde{\omega}\right|^{2}$ of the form

$$
\frac{d}{d t} \zeta(t)+\alpha(t) \zeta(t) \leq \beta(t)
$$

with

$$
\begin{gathered}
\alpha(t)=\min \left\{\alpha_{1}(t), \alpha_{2}(t)\right\} \\
\beta(t)=2 c\left|P_{m} \omega\right|_{g}^{1 / 2}\left\|P_{m} \omega\right\|_{g}^{1 / 2}\|u\|_{g}\left|Q_{m} \omega\right|_{g}^{1 / 2}\left\|Q_{m} \omega\right\|_{g}^{1 / 2} \\
+c\left|P_{m} \omega\right|_{g}^{1 / 2}\left\|P_{m} \omega\right\|_{g}^{1 / 2}\|\theta\|_{g}\left|\tilde{Q}_{m} \tilde{\omega}\right|_{g}^{1 / 2}\left\|\tilde{Q}_{m} \tilde{\omega}\right\|_{g}^{1 / 2} \\
+c\left|P_{m} \omega\right|_{g}^{1 / 2}\left\|P_{m} \omega\right\|_{g}^{1 / 2}\|\bar{\theta}\|_{g}\left|\tilde{Q}_{m} \tilde{\omega}\right|_{g}^{1 / 2}\left\|\tilde{Q}_{m} \tilde{\omega}\right\|_{g}^{1 / 2} \\
+2 c|\bar{u}|_{g}^{1 / 2}\|\bar{u}\|_{g}^{1 / 2}\left|P_{m} \omega\right|_{g}^{1 / 2}\left\|P_{m} \omega\right\|_{g}^{1 / 2}\left\|Q_{m} \omega\right\|_{g} \\
+c|u|_{g}^{1 / 2}\|u\|_{g}^{1 / 2}\left|\tilde{P}_{m} \tilde{\omega}\right|_{g}^{1 / 2}\left\|\tilde{P}_{m} \tilde{\omega}\right\|_{g}^{1 / 2}\left\|\tilde{Q}_{m} \tilde{\omega}\right\|_{g} \\
+c|\bar{u}|_{g}^{1 / 2}\|\bar{u}\|_{g}^{1 / 2}\left|\tilde{P}_{m} \tilde{\omega}\right|_{g}^{1 / 2}\left\|\tilde{P}_{m} \tilde{\omega}\right\|_{g}^{1 / 2}\left\|\tilde{Q}_{m} \tilde{\omega}\right\|_{g} \\
+\frac{2 \nu}{m_{0}}|\nabla g|_{\infty}\left\|P_{m} \omega\right\|_{g}\left|Q_{m} \omega\right|_{g}+\frac{2 \kappa}{m_{0}}|\nabla g|_{\infty}\left\|\tilde{Q}_{m} \tilde{\omega}\right\|_{g}\left|\tilde{P}_{m} \tilde{\omega}\right|_{g} \\
+2|\xi|_{\infty}\left|\tilde{P}_{m} \tilde{\omega}\right|_{g}\left|Q_{m} \omega\right|_{g}+2\left\|f_{1}-\bar{f}_{1}\right\|_{V_{g}^{\prime}}\left\|Q_{m} \omega\right\|_{g}+2\left\|f_{2}-\bar{f}_{2}\right\|_{W_{g}^{\prime}}\left\|\tilde{Q}_{m} \tilde{\omega}\right\|_{g}
\end{gathered}
$$

where

$$
\begin{aligned}
& \alpha_{1}(t)=\nu \lambda_{m+1}-\left[\frac{5 c^{2}}{\nu}\|\nabla u\|_{g}^{2}+\frac{5 \nu}{m_{0}^{2}}\|\nabla g\|_{\infty}^{2}+\frac{5 c^{4}}{2 \nu}\right] \\
& \alpha_{2}(t)=\kappa \lambda_{m+1}-\left[\frac{3}{64 \kappa}\left(\|\nabla \theta\|_{g}^{4}+\|\nabla \bar{\theta}\|_{g}^{4}\right)+\frac{5 M_{0}}{4 \pi^{2} \nu m_{0}}\|\xi\|_{\infty}^{2}+\frac{3 \kappa}{m_{0}^{2}}\|\nabla g\|_{\infty}^{2}\right]
\end{aligned}
$$

Since the solutions $u, \bar{u}, \theta$ and $\bar{\theta}$ are bounded uniformly for $t$ bounded away from zero in $H_{g}, V_{g}$ and $W_{g}$ respectively and by assumptions (4.9) and (4.10) it follows that $\beta(t) \rightarrow 0$ as $t \rightarrow \infty$. One can deduce the following inequality from (3.9) and (3.10) for sufficiently large $T$ :

$$
\begin{aligned}
\frac{1}{T} \int_{t}^{t+T}\|u(\tau)\|_{g}^{2} d \tau & \leq \frac{2 M_{0}^{2}|\xi|_{\infty}^{2}}{\nu^{\prime 2} \kappa^{\prime 2} \pi^{4} m_{0}^{2}}\left\|f_{2}\right\|_{L^{\infty}\left(t, t+T ; L^{2}(\Omega, g)\right)}^{2}+\frac{8}{\nu^{\prime 2}}\left\|f_{1}\right\|_{L^{\infty}\left(t, t+T ; H_{g}\right)}^{2} \\
\frac{1}{T} \int_{t}^{t+T}\|\theta(\tau)\|_{g}^{2} d \tau & \leq \frac{4}{\kappa^{\prime 2}}\left\|f_{2}\right\|_{L^{\infty}\left(t, t+T ; L^{2}(\Omega, g)\right)}^{2} .
\end{aligned}
$$

Then we see that

$$
\begin{array}{r}
\limsup _{t \rightarrow \infty} \frac{1}{T} \int_{t}^{t+T} \alpha_{1}^{-}(\tau) d \tau=\frac{5 c^{2}}{\nu}\left[\frac{2 M_{0}^{2}|\xi|_{\infty}^{2}}{\nu^{\prime 2} \kappa^{\prime 2} \pi^{4} m_{0}^{2}}\left\|f_{2}\right\|_{L^{\infty}\left(t, t+T ; L^{2}(\Omega, g)\right)}^{2}+\frac{8}{\nu^{\prime 2}}\left\|f_{1}\right\|_{L^{\infty}\left(t, t+T ; H_{g}\right)}^{2}\right] \\
+\frac{5 \nu}{m_{0}^{2}}|\nabla g|_{\infty}^{2}+\frac{5 c^{4}}{2 \nu}-\nu \lambda_{m+1}<\infty \\
\limsup _{t \rightarrow \infty} \frac{1}{T} \int_{t}^{t+T} \alpha_{2}^{-}(\tau) d \tau=\frac{3}{4 \kappa \kappa^{\prime 4}}\left[\left\|f_{2}\right\|_{L^{\infty}\left(t, t+T ; L^{2}(\Omega, g)\right)}^{4}+\left\|\bar{f}_{2}\right\|_{L^{\infty}\left(t, t+T ; L^{2}(\Omega, g)\right)}^{4}\right] \\
+\frac{5 M_{0}}{4 \pi^{2} \nu m_{0}}|\xi|_{\infty}^{2}+\frac{3 \kappa}{m_{0}^{2}}|\nabla g|_{\infty}^{2}-\kappa \lambda_{m+1}<\infty
\end{array}
$$


and the condition (4.2) of Gronwall lemma is satisfied. Finally, we see that

$$
\begin{array}{r}
\liminf _{t \rightarrow \infty} \frac{1}{T} \int_{t}^{t+T} \alpha_{1}(\tau) d \tau \geq \nu \lambda_{m+1}-\frac{5 \nu}{m_{0}^{2}}|\nabla g|_{\infty}^{2}-\frac{5 c^{4}}{2 \nu} \\
-\frac{5 c^{2}}{\nu}\left[\frac{2 M_{0}^{2}|\xi|_{\infty}^{2}}{\nu^{\prime 2} \kappa^{\prime 2} \pi^{4} m_{0}^{2}}\left\|f_{2}\right\|_{L^{\infty}\left(t, t+T ; L^{2}(\Omega, g)\right)}^{2}+\frac{8}{\nu^{\prime 2}}\left\|f_{1}\right\|_{L^{\infty}\left(t, t+T ; H_{g}\right)}^{2}\right] \\
\liminf _{t \rightarrow \infty} \frac{1}{T} \int_{t}^{t+T} \alpha_{2}(\tau) d \tau \geq \kappa \lambda_{m+1}-\frac{5 M_{0}}{4 \pi^{2} \nu m_{0}}|\xi|_{\infty}^{2}-\frac{3 \kappa}{m_{0}^{2}}|\nabla g|_{\infty}^{2} \\
-\frac{3}{4 \kappa \kappa^{\prime 4}}\left[\left\|f_{2}\right\|_{L^{\infty}\left(t, t+T ; L^{2}(\Omega, g)\right)}^{4}+\left\|\bar{f}_{2}\right\|_{L^{\infty}\left(t, t+T ; L^{2}(\Omega, g)\right)}^{4}\right]
\end{array}
$$

and if $m$ is sufficiently large that the inequalities

$$
\begin{aligned}
& \lambda_{m+1} \geq \frac{5 c^{2}}{\nu^{2}}\left[\frac{2 M_{0}^{2}|\xi|_{\infty}^{2}}{\nu^{\prime 2} \kappa^{\prime 2} \pi^{4} m_{0}^{2}}\left\|f_{2}\right\|_{L^{\infty}\left(t, t+T ; L^{2}(\Omega, g)\right)}^{2}+\frac{8}{\nu^{\prime 2}}\left\|f_{1}\right\|_{L^{\infty}\left(t, t+T ; H_{g}\right)}^{2}\right]-\frac{5}{m_{0}^{2}}|\nabla g|_{\infty}^{2}-\frac{5 c^{4}}{2 \nu^{2}} \\
& \lambda_{m+1} \geq \frac{3}{4 \kappa^{2} \kappa^{\prime 4}}\left[\left\|f_{2}\right\|_{L^{\infty}\left(t, t+T ; L^{2}(\Omega, g)\right)}^{4}+\left\|\bar{f}_{2}\right\|_{L^{\infty}\left(t, t+T ; L^{2}(\Omega, g)\right)}^{4}\right]-\frac{5 M_{0}|\xi|_{\infty}^{2}}{4 \pi^{2} \nu \kappa m_{0}}-\frac{3}{m_{0}^{2}}|\nabla g|_{\infty}^{2}
\end{aligned}
$$

are satisfied then we can apply Gronwall lemma to deduce that

$\zeta(t)=\left|Q_{m} \omega\right|^{2}+\left|\tilde{Q}_{m} \tilde{\omega}\right|^{2}$ goes to zero as $t$ goes to infinity.

\section{References}

[1] Bae, H., Roh, J., Existence of Solutions of the g-Navier-Stokes Equations, Taiwanese J. Math., 8, No. 1, 85-102, 2004.

[2] Boland, J. and Layton, W., Error analysis for finite element methods for steady natural convection problems, Numer. Funct. Anal. Optim., 11:5-6, 449-483, 1990, DOI: $10.1080 / 01630569008816383$.

[3] Galdi,G.P., Lectures in Mathematical Fluid Dynamics, Birkhäuser-Verlag, 2000.

[4] Farhat, A., Jolly, M.S., and Titi, E.S., Continuous Data Assimilation for the 2D Bénard Convection Through Velocity Measurements Alone, Physica D, 303, 59-66, 2015.

[5] Foias, C., Jolly, M.S., Kravchenko, R., and Titi, E.S., A unified approach to determining forms for the $2 D$ Navier-Stokes equations - the general interpolants case, Russ. Math. Surv.,69, No. 2, 359-381, 2014.

[6] Foias, C., Manley, O., Rosa, R. and Temam, R., Navier - Stokes Equations and Turbulence, Encyclopedia of Mathematics and Its Applications, vol. 83, Cambridge University Press, 2004.

[7] Foias, C., Manley, O., Temam, R., Attractors for the Bénard problem: Existence and physical bounds on their fractal dimension, Nonlinear Anal. Theory, Methods \& Applications, 11, 939-967, 1987.

[8] Foias, C., Prodi, G., Sur le comportement global des solutions non stationnaires des équations de Navier-Stokes en dimension two, Rend. Sem. Mat. Univ., Padova, 39, 1-34, 1967.

[9] Hale, J.K., Raugel, G., A damped hyperbolic equation on thin domains, Trans. Amer. Math. Soc., 329, 185-219, 1992.

[10] Hale, J.K., Raugel, G., Partial differential equations on thin domains, Differ. Eq. Math. Phys., Birmingham, AL, 1990, Academic Press, Boston, 63-97, 1992.

[11] Hale, J. K., Raugel, G., Reaction - Diffusion equation on thin domains, J. Math. Pures Appl., 71, 33-95, 1992.

[12] Hoang, L.T., Incompressible Fluids in Thin Domains with Navier Friction Boundary Conditions (I), J. Math. Fluid Mech., 12, No. 3, 435-472, 2010.

[13] Hoang, L.T., Incompressible Fluids in Thin Domains with Navier Friction Boundary Conditions (II), J. Math. Fluid Mech., 15, 361-395, 2013.

[14] $\mathrm{Hu}, \mathrm{C} .$, Navier-Stokes equations in $3 D$ thin domains with Navier friction boundary condition, J. Differ. Equ., 236, No. 1, 133-163, 2007.

[15] Hu, C., Global strong solutions of Navier-Stokes equations with interface boundary in threedimensional thin domains, Nonlinear Anal. 74, No. 12, 3964-3997, 2011. 
[16] Iftimie, D., The 3D Navier - Stokes equations seen as a perturbation of the 2D Navier Stokes equations, Bull. Soc. Math., France, 127, 473-517, 1999.

[17] Iftimie, D. and Raugel, G., Some results on the Navier-Stokes equations in thin $3 D$ domains, J. Differ. Equ., 169, 281-331, 2001.

[18] Iftimie, D., Raugel, G., Sell, G.R., Navier-Stokes equations in thin $3 D$ domains with Navier boundary conditions, Indiana Univ. Math. J., 56, No. 3, 1083-1156, 2007.

[19] Jones, D.A., and Titi, E.S., Determination of the solutions of the Navier - Stokes equations by finite volume elements, Phys. D, 60, 165-174, 1992.

[20] Jones, D., Titi, E.S., Upper bounds on the number of determining modes, nodes, and volume elements for the Navier - Stokes equations, Indiana Univ. Math. J., 42, No. 3, 875-887, 1993.

[21] Kagei, Y., On weak solutions of nonstationary Boussinesq equations, Diff. Integral Equ., 6 , 587-611, 1993.

[22] Kapustyan, O.V., Melnik, V.S., Valero, J., A weak attractor and properties of solutions for the three-dimensional Bénard problem, Discr. Contin. Dyn. Syst. Ser. A, 18, 449-481, 2007.

[23] Kapustyan, O.V., Pankov, A.V., Global $\varphi$-attractor for a modified 3D Bénard system on channel-like domains, Nonauton. Dyn. Syst., 1, Issue 1, 1-9, 2014.

[24] Kapustyan, O.V., Pankov, A.V., Valero, J., On global attractors of multivalued semiflows generated by the 3D Bénard system, Set-Valued and Variat. Anal., 20, 445-465, 2012.

[25] Kaya, M. and Çelebi, A.O., Existence of weak solutions of the g-Kelvin-Voight equation, Math. Comput. Modelling, 49, 497-504, 2009.

[26] Kaya, M. and Çelebi, A.O., Global attractor for the regularized Bénard problem, Appl. Anal., 93, Issue 9, 1989-2001, 2014.

[27] Ladyzhenskaya O.A., The Mathematical Theory of Viscous Incompressible Flow, 2nd Edition, Gordon and Breach, New York, 1969.

[28] Moise, I., Temam, R., Ziane, M., Asymptotic analysis of the Navier - Stokes equations in thin domains, Topol. Methods Nonlinear Anal., 10, 249-282, 1997.

[29] Montgomery, S., Global regularity of the Navier-Stokes equations on thin three-dimensional domains with periodic boundary conditions, Electron. J. Differ. Equ., 11, 1-19, 1999.

[30] Morimoto, H., Non-stationary Boussinesq equations, J. Fac. Sci. Univ. Tokyo Sect. IA Math, 39, 61-75, 1992.

[31] Olson, E., Titi, E.S., Determining modes for continuous data assimilation in 2D turbulence, J. Stat. Physics, 113, No.516, 799-840, 2003.

[32] Olson, E., Titi, E.S., Determining modes and Grashof number in 2D turbulence: a numerical case study, Theor. Comput. Fluid Dyn., 22, Issue 5, 327-339, 2008.

[33] Raugel, G., Sell, G.R., Navier - Stokes equations on thin $3 D$ domains. I. Global attractors and global regularity of solutions, J. Amer. Math. Soc., 6, 503-568, 1993.

[34] Raugel, G., Sell, G.R., Navier - Stokes equations on thin 3D domains. II. Global regularity of spatially periodic solutions, Nonlinear Partial Differential Equations and Their Applications, Collège de France Seminar, vol. XI, Longman, Harlow, , 205-247, 1994.

[35] Roh, J., g-Navier-Stokes equations, Thesis, University of Minnesota, 2001.

[36] Roh, J., Dynamics of the g-Navier-Stokes equations, J. Differ. Equ., 211, No. 2, 452-484, 2005.

[37] Roh, J., Geometry of $L^{2}(\Omega, g)$, J. Chungcheong Math. Soc., 19, No.3, 283-289, 2006.

[38] Temam, R., Navier-Stokes Equations and Nonlinear Functional Analysis, CBMS Regional Conference Series, No. 41, SIAM, Philadelphia, 1983.

[39] Temam, R., Navier-Stokes Equations, Theory and Numerical Analysis, vol. 2 of Studies in Mathematics and Its Applications, North-Holland, Amsterdam, The Netherlands, 3rd edition, 1984.

[40] Temam, R. and Ziane, M., Navier-Stokes equations in three-dimensional thin domains with various boundary conditions, Adv. Differ. Equ., 1, 499-546, 1996.

[41] Temam, R. and Ziane, M., Navier-Stokes equations in thin spherical domains, Contemp. Math., 209, 281-314, 1997.

[42] Wu, D., On the Dimension of the Pullback Attractors for g-Navier-Stokes Equations, Discrete Dyn. Nature Soc., 2010, Article ID 893240, 16 pages. 